\title{
Towards the experimental validation of a small Time-Projection-Chamber for the quasi-absolute measurement of the fission cross section
}

\author{
Carole Chatel ${ }^{1,2}$, Ludovic Mathieu $^{1}$, Mourad Aïche ${ }^{1}$, Maria Diakaki $^{2}$, Gilles Noguere $^{2}$ and \\ Olivier Bouland ${ }^{2}$ \\ ${ }^{1}$ Centre d'Etude Nucléaire de Bordeaux-Gradignan, FRANCE \\ ${ }^{2}$ CEA Cadarache, DES/IRESNE/DER/SPRC/LEPh, FRANCE \\ Corresponding author: carole.chatel@cea.fr
}

\begin{abstract}
To accurately measure neutron-induced fission cross sections, to characterize neutron-beam lines or to make dosimetric investigations, it is necessary to have high accuracy measurements of neutron fluence. It is possible to perform independent and precise neutron flux measurements with respect to the ${ }^{1} \mathbf{H}(\mathbf{n}, n) p$ elastic scattering cross section. The use of a silicon detector is recommended from 1 to $70 \mathrm{MeV}$ neutron energy. However, it has been observed that a high electrons background forbids its use below $1 \mathrm{MeV}$. Hence, a new gaseous proton-recoil telescope is developed and characterized to overcome this limit. It should provide quasi-absolute neutron flux measurements with an accuracy around $3 \%$ and is not sensible to gamma and electrons background. It consists in two ionization chambers read by a segmented micromegas technology detection plane. The gas pressure inside is adjustable to the proton range in the detector and therefore to the neutron energy. This detector is described in details below and the newest results of its characterization are presented. A special attention is paid to detection efficiency measurements.
\end{abstract}

Keywords - Gaseous proton recoil telescope, low energy neutron flux measurements.

\section{INTRODUCTION}

A BSOLUTE measurements of neutron fluence is a prerequisite for neutron-induced cross section measurements, neutron-beam lines characterization or dosimetric investigations. The development of the Generation IV nuclear systems (fast nuclear reactors) [1] or the accuracy improvement of the current neutronics code calculation require precise cross sections of neutron-induced reactions, mainly on heavy nuclei. However, despite the great general effort made by the nuclear community, discrepancies still exist between different measurements, especially in the actinides region.

To measure neutron-induced reaction cross sections, secondary standards $\left({ }^{235} \mathrm{U}(\mathrm{n}, \mathrm{f}),{ }^{238} \mathrm{U}(\mathrm{n}, \mathrm{f}),{ }^{237} \mathrm{~Np}(\mathrm{n}, \mathrm{f})\right)$ are usually used for the neutron flux measurements. These standards are measured with respect to a primary standard, so their accuracy ranges from 0.5 to $10 \%$ between 0 to $20 \mathrm{MeV}$. Moreover, the use of the same secondary creates correlations between the different measurements. An independent and precise technique of neutron-flux measurements becomes desirable. It is then possible to short-circuit secondary standards by using directly a primary standard. This can be performed with respect to the ${ }^{1} \mathrm{H}(\mathrm{n}, \mathrm{n}) \mathrm{p}$ elastic scattering cross section, known with an accuracy between $0.2 \%$ to $0.5 \%$ in a large energy range ( 0 to $20 \mathrm{MeV})$ [2, 3] and reproducible with ab initio calculations. This cross section has also the advantage of being monotonous. Hence, observed resonances are not correlated to standards' one. Silicon junction to make silicon-based proton-recoil telescope is the best choice to perform mono-energetic neutron flux measurements between 1 to $70 \mathrm{MeV}$ neutron energy [4, 5, $6,7]$. However, the maximum flux is between $100 \mathrm{keV}$ to few $\mathrm{MeV}$ in fast nuclear reactors and measurements at these low energies prevent the use of silicon detectors. Indeed, even if adapting silicon's thickness to neutrons energies is recommended, a high gamma and electrons background due to the neutrons' source prevent the use of a silicon below $1 \mathrm{MeV}$ [4]. This is because the energy of the electron deposited in the whole detector thickness in the silicon detectors becomes comparable to the energy of the proton which prohibits a clear discrimination between the protons' signal and the background.

Of course, other proton-recoil detectors have been developed. In this neutron energy range, plastic scintillators [8] are commonly used. Yet, the light produced by these detectors is not linear with neutron energy. Moreover, neutron reactions on the ${ }^{12} \mathrm{C}$ must be discriminated [9-12]. Finally, their detection efficiency cannot be accurately measured. Thus, these detectors are not suited to our needs. Proportional counter detectors also exist [13-15]. However, their efficiency must be rebuilt by simulation, along with other constraints (gas ionization potential, ionizing particle energy, edge-effects, interferences from photons and carbon recoils [13, 16 17]) which prohibit again their use for our needs.

Thus, it seems that no detector already exists to answer our needs. That is why the ACEN team of the CENBG decided to develop a gaseous proton-recoil telescope able to accurately measure neutron fluxes in the neutron energy range of 0.2 to 2 $\mathrm{MeV}$ and not sensitive to high gamma and electrons background. 


\section{DETECTOR DESCRIPTION}

The gaseous proton-recoil telescope (GPRT) is composed of two ionization chambers ( $\Delta \mathrm{E}$ and $\mathrm{E}$ ) separated by a collimator. The electric field is made by the presence of a cathode and a micromegas technology detector plane. This plane is composed by two electrodes: an anode segmented in 64 pads and a biased mesh $120 \mu \mathrm{m}$ above. A field cage homogenizes the electric field [18]. A macor disk to carry samples stands upstream the detector. A first collimator stands after and represents the entrance of the detector. A disposition of the detector is shown in figure 1 . A zoom on the detection plane is made in figure 2.

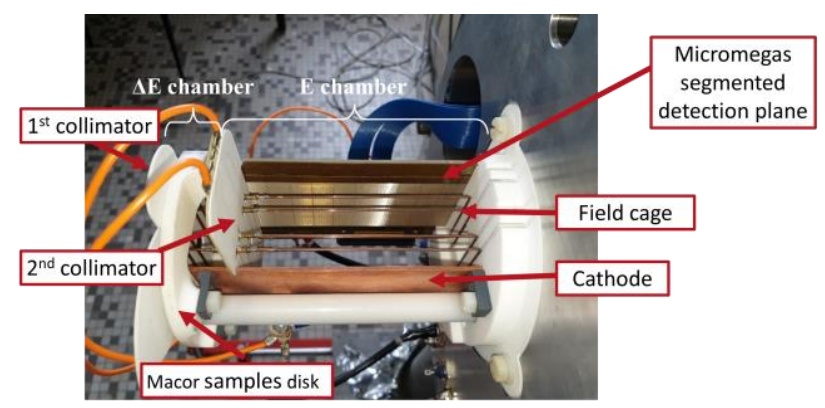

Figure 1: Picture of the Gaseous Proton-Recoil Telescope

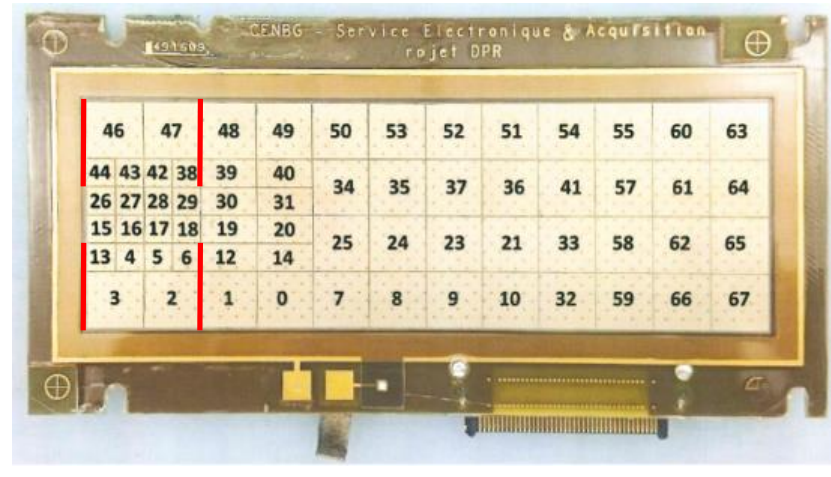

Figure 2: Micromegas detector plane. Red thick lines represent the places of both collimators ( $\Delta \mathrm{E}$ chamber)

\section{A. Choices made for the GPRT optimal functioning}

The macor disk can carry up to eight $\mathrm{H}$ foils. The aim is to adapt the foil thickness to the neutron energy so the energy loss of the recoil-proton is less than $10 \%$ in the foil. This prohibits a too big energy spread, which is essential to make accurate measurements.

Neutrons imping an $\mathrm{H}$ foil on the samples disk and recoil protons are collimated a first time before entering the detector to clearly define the sample active area. They are collimated a second time before being detected in the second chamber $(\mathrm{E}$ chamber). The aim is to limit the protons' angle so that their energy is similar to that of the impinging neutrons. The presence of these two collimators also well-defines the geometry, and thus the geometrical efficiency, and allows a physical separation between the two chambers. The detector plane is segmented in 64 pads that will each collect an individual signal. Thus, it is possible to check the proton's track. Correct proton tracks have to activate pads in the $\Delta \mathrm{E}$ chamber and in the E chamber and to have passed through both collimators. Parasitic tracks, for instance those induced by scattered neutrons, have to be discarded.

To reduce as much as possible the number of these unwanted protons, the collimators, as well as the structures, the samples disk and the detector chamber, are made of macor, a $\mathrm{H}$-free material and with as little material as possible to reduce neutrons scattering.

The chamber is filled with an $\mathrm{H}$-free gas. The one chosen is made of $70 \% \mathrm{~N}_{2}, 30 \% \mathrm{CO}_{2}$. We will discuss later this choice. The gas pressure is chosen so that protons are stopped in the detector. Thus, it is possible to see their Bragg's peak. Once the gas pressure is decided, the polarization voltage have to be applied. It has to be as high as possible to maximize the amplification gain. The breakdown voltages as a function of pressure for this gas have been investigated. To avoid microbreakdown occurring when operating near the breakdown voltage, it has then been decided to work $20 \mathrm{~V}$ below breakdown voltages.

\section{B. Acquisition}

The signal collected by the micromegas pads is treated by three successive electronics cards. The first one: the Zap card, is useful to protect the two other cards in case of breakdowns. The second card, called SAM for Single AGET Module, preamplifies the signal with four possible gains chosen by the user. A discriminator also receives the signal. The discriminator compares the signal level with the amplitude threshold and with the multiplicity threshold, both chosen by the user. This means that the signal will be registered only if the number of activated pads by the incoming particle is equal or higher than the number chosen by the user as triggers and that their amplitude is higher than the threshold. The last card, named ZedBoard, reduces the data size, sends events and timing to the computer. All the signal treatment last few nanoseconds per point and the dead time is of $20.5 \mu \mathrm{s}$ per channel, corresponding to $1.4 \mathrm{~ms}$ for the 64 pads channels.

Once treated, the signal is sent to the acquisition system. The software, named Cobo, has been developed by CEA Saclay. It allows the user to determine adjustable parameters as threshold, multiplicity, pads triggers and gain; to start and stop the acquisition and to visualize detected events.

Another software (CoboFrameViewer, developed at CEA Saclay) is used to read recorded data once stopped and saved. A Frame is composed of 64 curves, each corresponding to the response of one pad. An example is given in figure 3. 


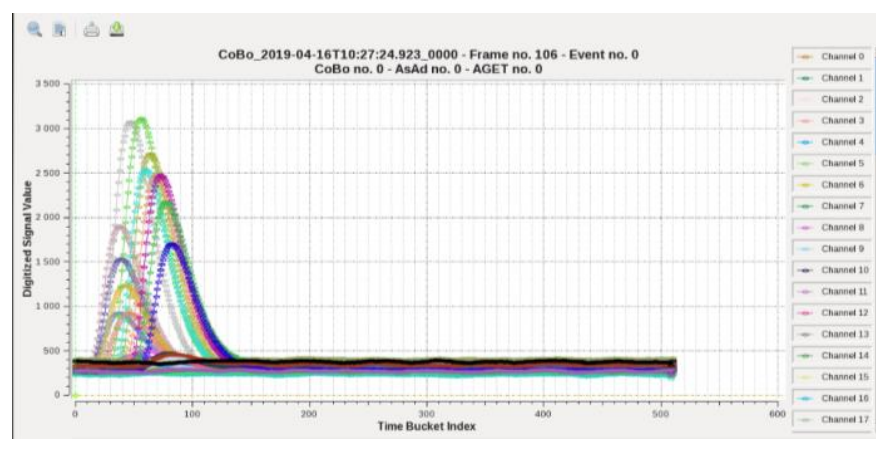

Figure 3: Frame obtained during an acquisition

\section{Tracks reconstruction and discrimination}

As each activated pad can be spotted by its curve, it is then possible to reconstruct the track accordingly to the activated pads. An example of a reconstructed track of an event is given in figure 4.

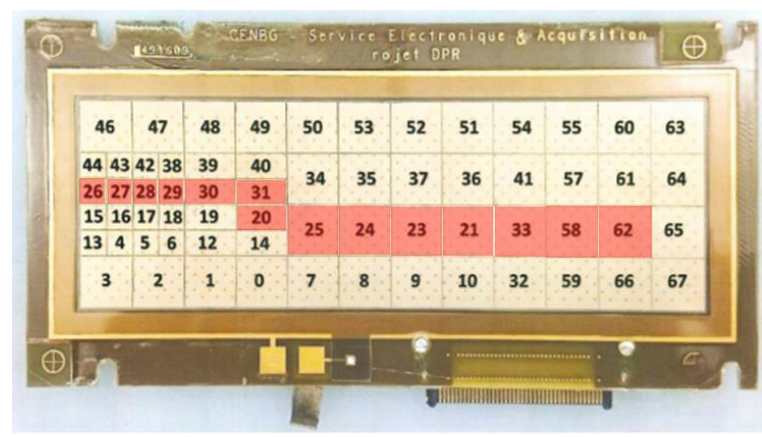

Figure 4: Example of a proton track reconstruction.

Reconstructing tracks is very useful to check the detected particle origin. Indeed, protons coming from scattered neutrons may not have a straight track passing through both collimators. Checking the $\Delta \mathrm{E}-\mathrm{E}$ coincidence is thus essential to discriminate and exclude from the analysis protons from scattered neutrons. Another possible but less convenient way to discriminate them is to compare the signal amplitude in frames. Indeed, a proton coming from a scattered neutron have a lower energy than a recoil proton and so has a shorter range. Its Bragg's pic is then before the one of a recoil proton. The track of a proton coming from a scattered neutron is presented in figure 5 (left). Sometimes, the GPRT detects other events coming from cosmics. Their tracks are easily recognized due to their vertical trajectory. The track of a cosmic ray is presented in figure 5 (right).
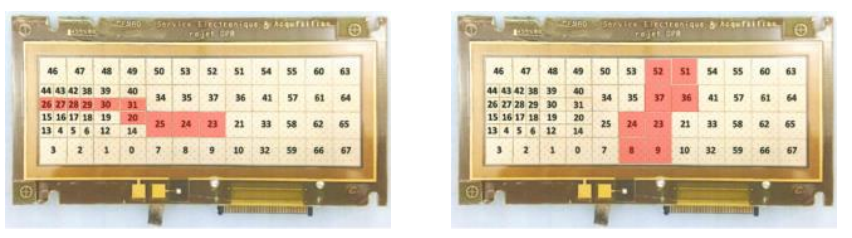

Figure 5: Reconstruction of the track of a proton coming from a scattered neutron (left) and of a cosmic ray (right)
Furthermore, it is possible to reconstruct the third dimension of tracks thanks to electrons drift velocity. Indeed, the chosen gas can be considered as a slow gas, namely electrons move slowly to join the anode. The drift velocity is quite constant at about $4 \mathrm{~cm} / \mu$ s for the different conditions used. Considering that a particle ionizes all the gas at the same time, electrons produced the closest of the detection plane arrive the first. The position shift of the different peaks (see Figure 3) allows to infer the arrival time via the sampling frequency (10 ns per point). For example, in the frame showed in Figure 3, the curves' maximum of the last pads in the segmented plane arrive in last so the particle got further from the detection plane as represented in figure 6.

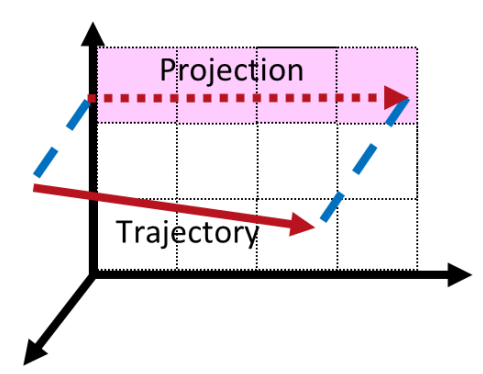

Figure 6: reconstruction of the $3^{\text {rd }}$ dimension thanks to the electron drift velocity.

Background tracks from $\gamma$-rays or electrons has also been investigated. As long as the threshold was suitable for proton detection, no unwanted-even frame was detected. One can deduce that the GPRT is non-sensitive neither to $\gamma$-rays nor to electrons, once proper trigger and threshold are set for protons.

Once the recoil-protons tracks can be discriminated from parasitic events, it is necessary to count them to make accurate measurements. In fact, any recoil-proton entering the detector must be detected to reduce as much as possible the uncertainties. This means that the detector's intrinsic efficiency must be equal to $100 \%$. Now that the device, acquisition and tracks reconstruction has been described, the determination of the GPRT's efficiency is described in the following section.

\section{DETECTION EFFICIENCY MEASUREMENTS}

First, the detection efficiency had been measured with an alpha source. Then, a mono-energetic proton micro-beam has been used to verify the results and extend them to other energies and other counting rates.

\section{A. Intrinsic efficiency with an alpha source}

The intrinsic efficiency depends of two other terms: the geometric efficiency and the detection efficiency as shown in equation (1):

$$
\varepsilon_{\text {intrinsic }}=\frac{\varepsilon_{\text {detection }}}{\varepsilon_{\text {geometric }}}
$$

In the case of the use of a source, the detection efficiency is 
equation (2)

$$
\varepsilon_{\text {detection }}=\frac{N}{A_{\text {source }} * t}
$$

With $\mathrm{N}$, the number of events detected during an acquisition, $\mathrm{t}$ the time of this acquisition and $\mathrm{A}_{\text {source }}$ the activity of the alpha source. Hence, the geometric efficiency has to be calculated and the source activity has to be accurately measured before running an acquisition of a precise time.

The geometric efficiency was calculated by means of a Monte Carlo simulations. The parameters to calculate the GPRT geometric efficiency with the error are presented in Table I. The sensitivity of the geometric efficiency to each parameter has been investigated to infer the final uncertainties and is also presented in Table I. The same effort has been made to define the alpha activity uncertainty.

TABLE I

PARAMETERS TO CALCULATE THE GPRT GEOMETRIC EFFICIENCY

\begin{tabular}{cccc}
\hline \hline Parameters & $\begin{array}{c}\text { Values } \\
(\mathrm{mm})\end{array}$ & $\begin{array}{c}\text { Absolute } \\
\text { uncertainties }(\mathrm{mm})\end{array}$ & $\begin{array}{c}\text { Sensitivity } \\
(\% / \mathrm{mm})\end{array}$ \\
\hline Collimator radius & 5.00 & 0.01 & 38.91 \\
Source radius & 4.10 & 0.25 & 0.82 \\
$\begin{array}{c}\text { Distance source- } \\
\text { collimator }\end{array}$ & 26.2 & 0.5 & 7.33 \\
\hline \hline
\end{tabular}

The geometric efficiency of the GPRT device is $(0.87 \pm 0.03) \%$.

The alpha activity $\mathrm{A}_{\text {source }}$ was measured by a dedicated alpha spectrometry. A simple device with the source and a silicon detector was used to reach this goal. Silicon detectors are known to have an intrinsic efficiency of $100 \%$. The detector was placed as far as possible to minimize the geometric efficiency error. This reduces the statistics that was compensated by a longer run. As before, Monte-Carlo simulations were used to estimate the geometric efficiency of this setup.

The geometric efficiency of the device to measure the alpha source activity is $(0.0947 \pm 0.0008) \%$ for a measured activity of $\left(517.9 \pm 4.4^{\text {sys }} \pm 1.9^{\text {stat }}\right) \mathrm{Bq}$.

Knowing the alpha source activity and the GPRT geometric efficiency, acquisitions were performed to infer the intrinsic efficiency.

To run acquisitions, parameters introduced in part I.A. must be defined. Even if the alpha energies are much higher than foreseen recoil protons energies, we still want to be representative of the intended use of the GPRT. Thus, the chosen pressure is 100 mbar and the voltage is $-2000 \mathrm{~V}$ for the cathode and $-430 \mathrm{~V}$ for the electrified mesh. Intrinsic parameters of the firmware must also be defined. To stay in consistency with the intended use, the chosen triggers are the three columns after the second collimator. To be in optimal conditions, the multiplicity is one so a frame is registered if at least one of the pads of these three columns is activated. A multiplicity of two has also been tested. Tests have been done with different gains and different thresholds in order to optimize the intrinsic efficiency and the rate of parasitic events to reject.

Optimal conditions appeared to be with the highest gain and a multiplicity of one. The intrinsic efficiency is then $\left(99.2 \pm 3.7^{\text {sys }} \pm 1.1^{\text {stat }}\right) \%$ for 4.5 alphas per second passing through the second collimator. This result is compatible with the expected $100 \%$. However, the uncertainties are large. We can see that the limiting factor is the detector geometry that will have to be improved in the next version of the prototype. Tests proved that the threshold does not influence the efficiency for such high-energy particles. However, a higher multiplicity or a smaller gain decrease significantly the efficiency. The hardware, firmware and software response to these parameters must further be investigated.

Now that one knows the intrinsic efficiency with optimal conditions and with an alpha source, the use of a proton beam is required to check the results.

\section{B. Intrinsic efficiency with a proton beam}

The proton beam was performed by the AIFIRA facility (Bordeaux, France). The typical current at such facilities is few nA (i.e. $10^{10}$ protons/s) $[19,20]$ for classic analysis but can be tuned from $50 \mu \mathrm{A}$ to few ions/s depending on the purpose of the experiment and the chosen beamline. For this experiment, we need only few protons/s. Thus, the beam was defocused and passed through a micro collimator with a diameter of $20 \mu \mathrm{m}$.

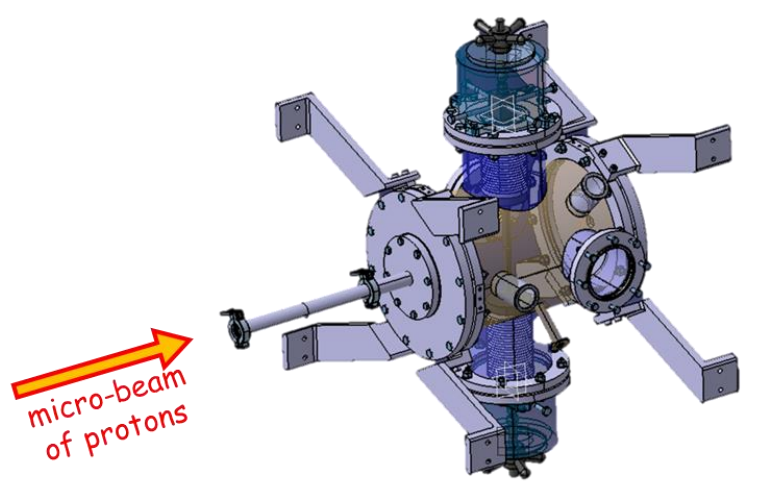

Figure 7: Scheme of the designed chamber for this experiment.

For this experiment, a dedicated chamber has been designed. This chamber is schematically shown in Figure 7. It has been designed to answer many goals. First of all, the chamber must be connected to the AIFIRA beam line. A window separates the accelerator vacuum from the gas filled chamber. The GPRT fits in the chamber, is connected to the electronics cards and aligned with the beam. As the number of protons entering the detector must be low, a collimator is placed at the entrance of the chamber and a second is before the entrance of the detector (to stop scattered protons from the first collimator). A set-up has also been designed so the GPRT can be moved inside the chamber to send the beam in different places of the detector (for 
instance, to test its spatial resolution). Finally, at the end of this chamber, a silicon detector can be fixed and connected so it will count the number of protons passing through the GPRT. All this device allows a much more precise analysis of the behavior and of the reproducibility of events than with an alpha source thanks to a mono-directional and mono-energetic protons beam.

The main test was to check the detector efficiency. The protons were delivered with an energy of $3 \mathrm{MeV}$. A silicon detector was placed downstream as explained above. Acquisitions were run with the GPRT optimal conditions mentioned above and the number of particles per second ranged from 0.6 protons/s to 366 protons/s. The gas pressure used was $100 \mathrm{mbar}$. At this pressure, the energy deposition of $3 \mathrm{MeV}$ protons is similar to that of low energy recoil protons. The efficiency resulted to be $100 \%$ for less than 3 protons/second. Above this value, the efficiency decreased to reach $7 \%$ for a beam of 366 protons/s. The $50 \%$ efficiency corresponded to 38 protons/s. This means that the GPRT seems to have an effective dead time of $26 \mathrm{~ms}$, much higher than the theoretical $1.4 \mathrm{~ms}$ presented in section I.B. However, these results are still coherent with the efficiency measured with the low activity alpha source.

A second set of tests was then performed. The silicon detector was there taken as a trigger for the GPRT. With these conditions, one can expect to overcome every GPRT issue and to register as many events as the silicon detector. However, the results were very similar to the first test. An investigation is currently carried out but the problem seems to come from the hardware or the software.

\section{CONCLUSIONS}

The Gaseous Proton Recoil Telescope is a miniaturized Time Projection Chamber developed at the CENBG (Bordeaux, France). This detector is intended to accurately measure a neutrons flux for fission cross sections measurements.

The GPRT characterization has been investigated with an alpha source and a direct protons beam. It appeared that the GPRT has an intrinsic efficiency equal to $100 \%$ with optimal initial conditions and with less than few particles per second. However, this efficiency falls when more restrictive conditions are used or when the number of particles becomes too high.

Further investigations are currently carried out to solve the efficiency issue. A special attention will be paid to the hardware and the software. Finally, once these problems are solved, another experiment will be planned on the AIFIRA facility to check the response of the detector with many different conditions.

\section{ACKNOWLEDGMENT}

The authors would like to acknowledge the AIFIRA staff for their help to realize and manage the experiment as well as their ideas to make better tests and to get better results.

\section{REFERENCES}

[1] NEA, "Nuclear data high priority request list", 2018
[2] J.C. Hopkins, G. Breit, "The ${ }^{1} \mathrm{H}(\mathrm{n}, \mathrm{n}){ }^{1} \mathrm{H}$ scattering observables required for high-precision fast-neutron measuremenst", Nuclear Data Tables A 9137,1971

[3] G.M. Hale, et al., Proc. $17^{\text {th }}$ Int. IUPAP, conf. on few-body problems in physics, Elsevier, Amsterdam pp S120-S122, 2004

[4] P. Marini, et al., "Systematic investigation of background sources in neutron flux measurements with proton-recoil silicon detector", Nuclear Instruments and Methods in physics A 841 56-64, 2016

[5] P. Marini, et al., "242 Pu neutron-induced fission cross section measurement from 1 to $2 \mathrm{Mev",} \mathrm{Physical} \mathrm{Review} \mathrm{C} \mathrm{96,} 054604$ (2017)

[6] S.J.Bame Jr, et al., "Absolute determination of monoenergetic neutron flux in the energy range 1 to $30 \mathrm{MeV}$ ", Review of Scientific Instruments 28, 997, 1957

[7] T. Ryves, Nuclear Instruments and Methods in physics 135, 455, 1976

[8] R. Beyer, et al., "Proton-recoil detectors for time-of-flight measurements of neutrons with kinetic energies from some tens of keV to a few $\mathrm{MeV}$," Nuclear Instruments and Methods in physics A 575, 449, 2007

[9] M.W. Wu, et al., "Absolute neutron fluence measurements between 0.5 and $3 \mathrm{MeV}$ and their intercomparisons", Nuclear Instruments and Methods in physics A 422, 79, 1999

[10] B. Daub, et al., "Response of BC-418 plastic scintillator to low energy protons", Nuclear Instruments and Methods in physics A 701, 171, 2013

[11] M. Kovash, et al., ANIMMA 2011, PP 1-3, 2011

[12] D. Schmidt, et al., "Characterization of liquid scintillation detectors", Nuclear Instruments and Methods in physics A 476, 186, 2002

[13] T.H.R. Skyrme, et al., "A proportional counter for neutron flux measurements in the energy range 0.1 to $1 \mathrm{MeV}^{\prime}$, Review of Scientific Instruments 23, 204, 1952

[14] E.F. Bennett, "Proportional counter proton-recoil spectrometer with gamma discrimination", Review of Scientific Instruments 33, 1153, 1962

[15] E.F. Bennett, T.J. Yule, “A neutron spectrum map of the JANUS irradiation facility using proton-recoil proportional counters", Radiation Research society 50, 219, 1972

[16] R. Babut, V Gressier, "(n,p) reactions contribution in spherical proton recoil proportional counter responses", Journal of Instrumentation 2, P01005, 2007

[17] R. Nolte, "Detection of neutrons", Ecole Joliot Curie 2014, 2014

[18] P. Marini, et al., "Development of a Gaseous Proton-Recoil Detector", WONDER-2018, 2018

[19] P. Barberet, et al., "AIFIRA : a light ion beam facility for ion beam analysis and irradiation", the European Physical Journal Plus, 2020

[20] S. Sorieul, P. Alfaurt, L. Daudin, L. Serani, P. Moretto, Nuclear Instruments and Methods in Physics Research Section B: Beam Interactions with Materials and Atoms 332, 68 (2014) 